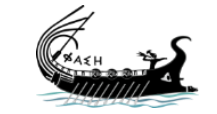

journal.phaselis.org
PHAS LLIS

Issue IV (2018)
Disiplinlerarası Akdeniz Araştırmaları Dergisi

Journal of Interdisciplinary Mediterranean Studies

\title{
Ein neuer Hausaltar aus Metropolis für Hadrian mit den Epitheten Olympios und Zeus Soter Augustus
}

\author{
Metropolis'ten Hadrianus İçin Olympios ve Zeus Soter \\ Augustus Epithetleriyle Yeni Bir Ev Sunağı
}

\section{Onur GÜLBAY}

The entire contents of this journal, Phaselis: Journal of Interdisciplinary Mediterranean Studies, is open to users and it is an 'open access' journal. Users are able to read the full texts, to download, to copy, print and distribute without obtaining the permission of the editor and author(s). However, all references to the articles published in the e-journal Phaselis are to indicate through reference the source of the citation from this journal.

Phaselis: Journal of Interdisciplinary Mediterranean Studies is a peer-reviewed journal and the articles which have had their peer reviewing process completed will be published on the web-site (journal.phaselis.org) in the year of the journal's issue (e.g. Issue III: JanuaryDecember 2017). At the end of December 2017 the year's issue is completed and Issue IV: January-December 2018 will begin.

Responsibility for the articles published in this journal remains with the authors.

Citation O. Gülbay, "Ein neuer Hausaltar aus Metropolis für Hadrian mit den Epitheten Olympios und Zeus Soter Augustus". Phaselis IV (2018) 231-237.

http://dx.doi.org/10.18367/Pha.18015

Received Date: 06.07.2018 | Acceptance Date: 14.09.2018

Online Publication Date: 17.10.2018

Editing Phaselis Research Project

www.phaselis.org 


\title{
Ein neuer Hausaltar aus Metropolis für Hadrian mit den Epitheten Olympios und Zeus Soter Augustus
}

\author{
Metropolis'ten Hadrianus İçin Olympios ve Zeus Soter Augustus Epithetleriyle \\ Yeni Bir Ev Sunağı
}

\author{
Onur GÜLBAY*
}

\begin{abstract}
Zusammenfassung: In Metropolis sind bisher die Kulte von Zeus Soter, Zeus Krezimos, Ares, Hera, die zwölf Götter, Dioskuren und Nymphai bekannt. Von all diesen Kulten ist das Heiligtum von Zeus Krezimos das einzige, das in Metropolis im Jahr 2015 archäologisch entdeckt wurde. Unter den beschränkten Zeugnissen über den Kaiserkult in der Stadt sind die dem Kaiser Hadrian gewidmeten Hausaltäre bekannt. Auf diesen Altären wurde Hadrian als ein olympischer Gott, Gründer und Retter verehrt. Neben Hadrian wurde auf einem Altar die Verehrung seiner Gattin Sabina mit Hera festgestellt. Im Ausgrabungssaison des Jahres 2018 bei den Bauarbeiten in Yeniköy, die sehr nahe an der Stadt liegt, ist ein Hausaltar mit zwei Inschriften gefunden worden, die auf die Assimilation von Hadrian mit Zeus hinweisen. Die erste Inschrift ist eine Weihung, auf der der Name Hadrians im Dativ angegeben ist und der Kaisar mit Zeus Olympios assimiliert wurde. Die zweite Inschrift ausdrückt, dass der Altar dem Zeus Soter Augustus gehört. Damit beweist sie zum ersten Mal, dass man Kaiser Hadrian wie in anderen Städten auch in Metropolis als Zeus Soter verehrte. Durch die Assimilation mit Zeus versuchte Hadrian zu zeigen, dass der Göttervater die Quelle seiner Macht bildete. Hierbei war es Hadrians Ziel, zu betonen, dass er die Politik seines Vorgängers der Annexion und der Romanisierung ablehnte und stattdessen die Idee der Koexistenz von griechischen und römischen Traditionen für das Wohlergehen des römischen Reiches förderte.
\end{abstract}

Schlüssel Wörter: Hausaltar, Hadrian, Zeus Soter, Sabina, Zeus Krezimos

Öz: Metropolis'te Zeus Soter, Zeus Krezimos, Ares, Hera, Oniki Tanrı, Dioskurlar ve Nympheler'in farlı epithetlerle tapınım gördükleri bilinmektedir. Bunlar içerisinde sadece Zeus Krezimos'un 2015 yılında keşfedilen kutsal alanı bilinmektedir. Metropolis'te imparator kültüyle ilgili sınırlı sayıda belge arasında Hadrianus'a adanmış birkaç ev sunağı bilinmektedir. Bu sunaklarda Hadrianus Olympios, ktistes ve soter epithetlerini taşımaktadır. Bir başka sunak ise eşi Sabina'nın Hera ile ortak bir külte sahip olduğunu belgelemektedir. 2018 sezonu içerisinde antik kente çok yakın bir konumda olan Yeniköy'deki bir inşaat yıkımı çalışmasında ele geçen bir ev sunağı Hadrianus kültünün Zeus ile özdeşleştirildiği iki yazıt taşımaktadır. Hadrianus' un dativus halinde verildiği ve Zeus Olympios'la özdeşleştrildiği ilk yazıt adak yazıtı özelliği taşımaktadır. İkinci yazıt ise sunağın Zeus Soter Augustus'a ait olduğunu dile getirmekte ve böylece Hadrianus Augustus'un diğer kentlerde olduğu gibi Metropolis'te de Zeus Soter olarak tapınım gördüğünü ilk defa kanıtlamaktadır. Hadrianus kendini Zeus ile özdeşleştirmek suretiyle, tanrıların babasını iktidarının kaynağı olarak göstermeye çalışmışır. Bu noktada Hadrian'ın amacı haleflerinin ilhak ve Romalılaştırma poltikasını reddettiğini ve bunun yerine imparatorluğun selameti açısından Yunan ve Roma geleneklerinin bir arada varlığı düşüncesini desteklediğini vurgulamaktı.

Anahtar Kelimeler: Ev altarı, Hadrianus, Zeus Soter, Sabina, Zeus Krezimos

* Dr., Dokuz Eylül Üniversitesi, Edebiyat Fakültesi, Arkeoloji Bölümü, İzmir. onur.gulbay@deu.edu.tr 
Fundinventar: Kd-18-38

H.: $54 \mathrm{~cm}$; Dm.: $35 \mathrm{~cm}$; Bh.: 2,5-3,0 cm

a)

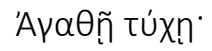

2 Аútокра́торь

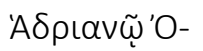

$4 \lambda$ $\lambda u \mu \pi i \omega \sigma \omega-$

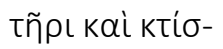

6 tก.

b)

$\Delta$ เòs

$2 \sum \omega \tau \tilde{\rho} \rho \circ \varsigma$

$\Sigma \varepsilon \beta \alpha \sigma \tau o u ̃$.

Die Zeugnisse über die Kultgeschichte der Stadt Metropolis sind beschränkt. Nur sind die Kulte von Zeus Soter, Zeus Krezimos, Hera, Apollon Pengasites, Ares, die zwölf Götter, Dioskuren und Nymphai bekannt ${ }^{1}$. Von all diesen Kulten ist das Heiligtum von Zeus Krezimos das einzige, das in Metropolis im Jahr 2015 archäologisch entdeckt wurde ${ }^{2}$. Unter den beschränkten Zeugnissen
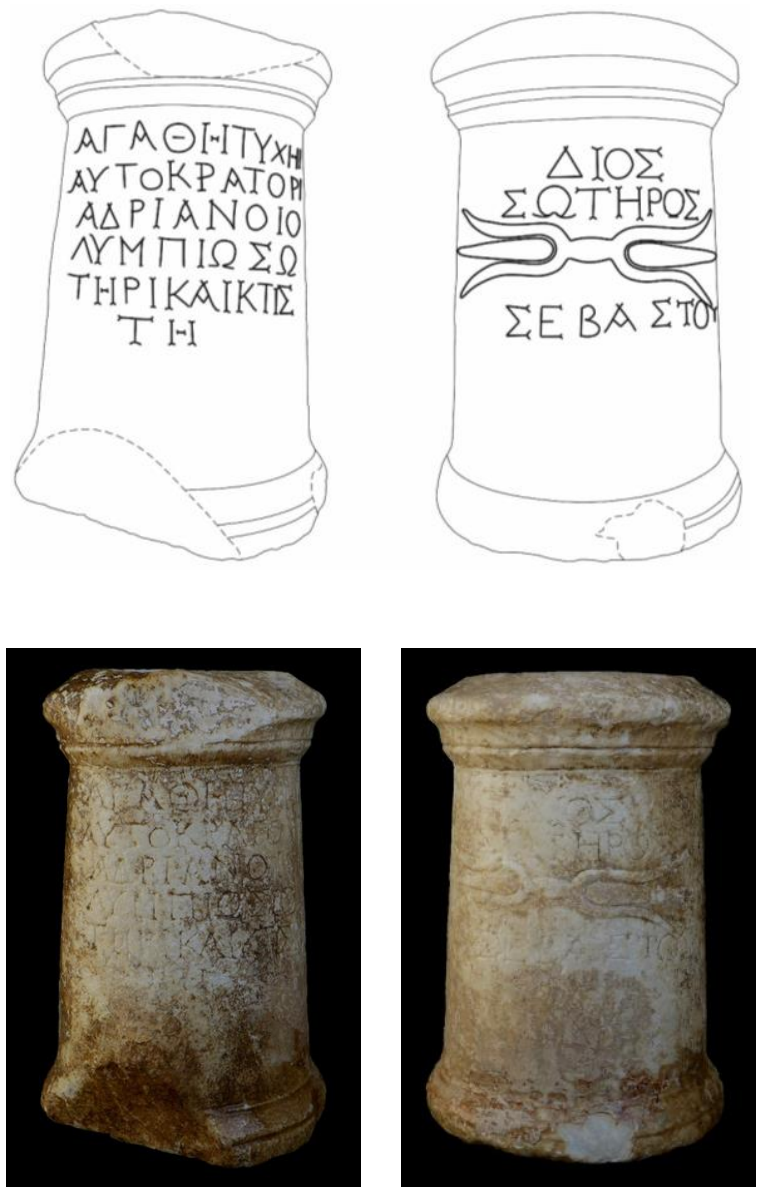
über den Kaiserkult in der Stadt sind die dem Kaiser Hadrian gewidmeten Hausaltäre bekannt ${ }^{3}$. Hadrian wurde im gesamten griechischen Osten mit Zeus identifiziert, wie in Benjamins Sammlung von Inschriften zu sehen ist ${ }^{4}$. Die Epitheta, die inn in seiner Identifikation mit Zeus begleiteten, beweisen die große Vorstellungskraft und die Bereitschaft der Griechen, ihn in ihren Widmungen und öffentlichen Aufzeichnungen als eine Manifestation des großen Gottes zu sehen: Er war Zeus Epikarpios in Korykos; ${ }^{5}$ Panhellenios in Ephesos, Pergamon, Miletos, Thyateira, lasos, Aphrodisias, Theos, Stratonikeia und Aizanoi; ${ }^{6}$ Panionios in Ephesos, Klaros und Teos; ${ }^{7}$ Olympios im ganzen Osten, in

1 Zeus Soter: IEphesos 3402; Zeus Kerazimos: IEphesos 3414-3415; Hera: IEphesos 3405, 3416; Apollon Pengasites: IEphesos 3406; Ares: IEphesos 3417; die zwölf Götter: IEphesos 3418; Dioskuren: IEphesos 3403; Nymphen: IEphesos 3404.

2 Aybek - Dreyer - Sponsel 2018, 71-94; vgl. Aybek - Gülbay 2018, 1-18.

3 Bei den in Metropolis und in ihrer Umgebung durchgeführten Untersuchungen kamen insgesamt drei unpublizierten Hausaltäre zutage. Für ein andere Beleg zur Verehrung des Hadrians als Zeus Olympios in Metropolis s. IEphesos 3410. Für solche Hausaltäre zu Ehren von Hadrian s. Witulski 2007, 130ff.

4 Benjamin 1963, 58; vgl. Baz 1998, 63-65; Price 2004, 258.

5 CIG 4433.

6 Ephesos: IEphesos 1501; Pergamon: IPergamon III 7; Miletos: IMilet 233-234; Thyateira: Jones 1999 ( = AE

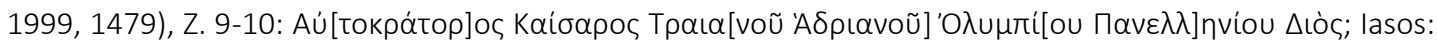
SEG 36,987a; Aphrodisias: Mendel 1906, 167; Teos: Robert 1946, 86-89; Stratonikeia: IGR IV 519; Aizanoi: SEG 42, 1191.

7 Ephesos: IEphesos 1501; Klaros: SEG 51, 1598; Teos: Robert 1946, 86-89 
griechischen Städten und römischen Kolonien gleichermaßen; Dodonaios in Nikopolis; ${ }^{8}$ Kynegesios in Stratonikeia- Hadrianopolis; ${ }^{9}$ er war sogar der Sohn von Trajan Zeus Eleutherios in Athen $^{10}$. Hadrian wurde oft gemeinsam mit seiner Frau Sabina (in Gestalt einer neuen Hera) geehrt, so zum Beispiel bei Philippi, Thasos, Metropolis, Patara, Tlos, Phaselis ${ }^{11}$.

Kaiser Hadrian galt bereits vor den Augen des Volkes ein Gott ${ }^{12}$ aber nachdem er im Jahr 128/9 n. Chr. das Epithet Olympios nahm, war seine Angleichung an Zeus offiziell geworden ${ }^{13}$. Hadrian kümmerte sich sowohl in Griechischen Osten um die Verehrung des Zeus. Er vervollständigte die früher begonnenen Zeustempel ${ }^{14}$. Das Denkmal, das am meisten zur Schaffung des Bildes von Hadrian als Zeus beigetragen hat, war das Heiligtum von Zeus Olympios in Athen ${ }^{15}$. Neben dem Olympieion begann Hadrian mit dem Bau von zwei weiteren Gebäuden, einem Tempel der Hera und Zeus Panhellenios und einem zweiten für alle Götter, einem Pantheon ${ }^{16}$. Alle drei Gebäude sind mit dem von Hadrian in 131/2 neu gegründeten Panhellenischen Bund verbunden. Indem er den Beinamen Olympios annahm, betonte Hadrian einerseits sein Panhellenisches Programm, denn Zeus Olympios, Hauptgottheit der Griechen, ist der Panhellenische Gott. Indem er den Beinamen Panhellenios erhält, wird er andererseits zur Macht, die über das Gemeinwesen der griechischen Städte herrscht, denn er ist der Zeus aller Griechen. Das römische Reich erlebte tatsächlich unter seiner Herrschaft wirtschaftlich und militärisch einen Höhepunkt. Zudem wurden in hadrianischer Zeit den religiösen Rituellen eine grosse Bedeutung beigemessen ${ }^{17}$. Es ist bekannt, dass Hadrian im Jahr 123 und 129 n. Chr. in die Stadt Ephesos kam, die in der Nähe von Metropolis liegt ${ }^{18}$. Nach heutigem Erkenntnisstand

8 SEG 35, 674; SEG 37, 521; SEG 39, 528; SEG 43, 343.

9 SEG 27, 809.

10 IG II 3322.

11 Philippi: AE 1984, 818; Thasos: IG XIISuppl. 440; Metropolis: IEphesos 3411; Patara: TAM II 412; Tlos: TAM II 560; Phaselis: Tüner Önen 2013, $97 \mathrm{ff}$.

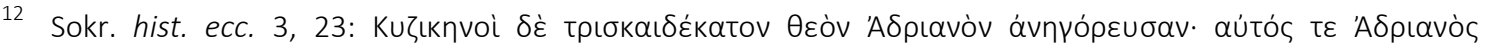

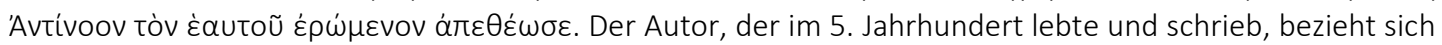
ohne Zweifel auf die Wohltaten des Kaisers für die Stadt, vor allem aber auf den Tempel, den Hadrian dem Volk von Kyzikos für seinen Kult erlaubte, so dass die Stadt zu seinen Neokoros wurde. Für Hadrians Anzeige bei der Thronbesteigung, dass er von Traian, seinem Adoptivvater, ernannt, aber von den Göttern ausgewählt wurde, s. POxy. 55, 3781.

13 Kienast 1990, 130; vgl. Gülbay 2009, 62. Er nahm den Beinamen Panhellenios seit Frühjahr 132 und den Beinamen Panionion nach 132, s. Kienast, loc. cit.

14 Burrell 2003, 31-50.

15 Der Tempel wurde 128 geweiht und die Fertigstellung kam fast vier Jahre später in 131/2, dem Jahr, in dem das Olympieion fertig war, die Statue von Olympios Zeus darin eingeweiht wurde, und das Panhellenion von Hadrian gegründet wurde. Polemon, der Sophist, wurde ausgewählt, um die Eröffnungsrede zu halten

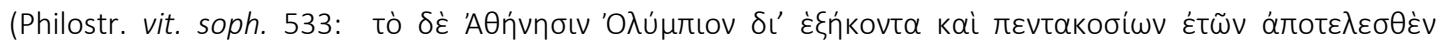

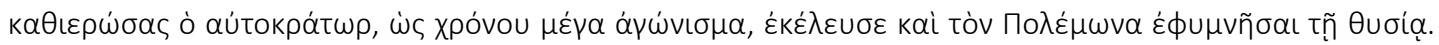

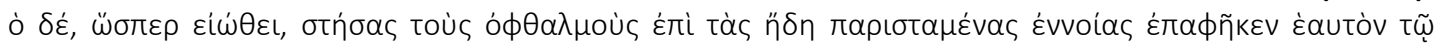
$\lambda$

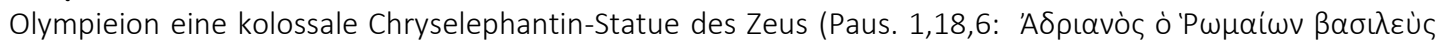

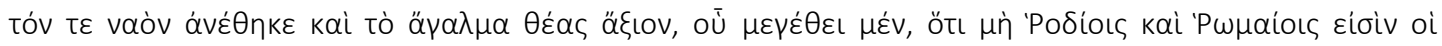

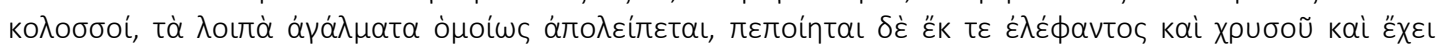

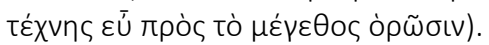

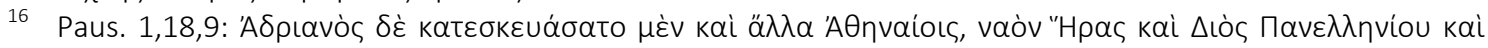

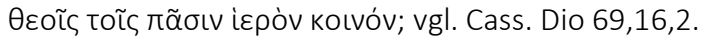

17 Walton 1957, 165-170.

18 Birley 1997, 222; Cadoux 2003, 332; Halfmann 1986, 188ff.; Wörrle 1988, 34f.; Kienast 1990, $128 f$. 
ist es nicht sicher, aber möglich, dass Hadrian während seiner Reise auch Metropolis besuchte ${ }^{19}$. Obwohl in manchen Bauten in Metropolis, zum Beispiel im Theater für Augustus, im oberen Bad für Nero, im unteren Bad für Antoninus Pius Widmungsinschriften festgestellt wurden ${ }^{20}$, ist in keinem antiken Gebäude eine Widmungsinschrift für Hadrian zu finden. Im Gegensatz zu den architektonischen Beweise zeigen die Altäre (s. oben Anm. 5), dass die Bürger der Stadt den Hadrian mit seinen olympischen Epitheten verehrten ${ }^{21}$. Das ist aber auf dem neuen Altar etwas neues zu sehen. Hier wurde Hadrian in Metropolis zum ersten Mal als "Zeus Soter" verehrt. Kurz nach der Assimilation mit Zeus und der Einnahme des Epithetons Olympios verwendete Hadrian auch den Beiname Soter. ${ }^{22}$ Auf einer Inschrift, die in der Nähe von Metropolis gefunden wurde, ist es festzustellen, dass die Metropoliten die Göttin Hera mit Sabina, die Gattin des Kaisers, verehrten $^{23}$. Damit wurden in Metropolis sowohl Hadrian-Zeus als auch Sabina-Hera miteinander gepaart und als gemeinsame Kulte verehrt. Auf dieser weise bezweckte man die Mitwirkung und Unterstützung der kaiserlichen Familien zum griechischen Pantheon.

Dank der Inschriften aus dem Heiligtum von Zeus Krezimos konnten über Prister und lokale Familien wichtige Details festgestellt werden ${ }^{24}$, die bei kultischen Rituellen mit Heiligtumsangestelltern tätig waren. Es gibt aber auffälliger weise keinen schriftlichen Nachweiss, dass irgendein Kaiser diesen Kult jemals unterstützte. Es könnte aus diesem Grund behauptet werden, während man Kaiser Hadrian mit dem Titel Zeus Soter nur auf den Hausaltäre verehrte, führten die lokalen Familien in Metropolis den Kult von Zeus Krezimos nur im Heiligtum auf. Damit anhand der in Metropolis entdeckten Hausaltäre ist zu verstehen, dass den Zeus Soter, den Kaiser Hadrian und in seinem Heligtum den Zeus Krezimos getrennt voneinander verehrt wurden ${ }^{25}$. Es ist unter anderem aus westlichen Kleinasien und dem griechischen Festland bekannt, dass die Kaiserkulte und Götterkulte zusammenausgeführt wurden ${ }^{26}$. Auf den bis jetzt in Metropolis gefundenen drei Hausaltären ist Hadrian mit den Titeln Retter und Gründer zu belegen. Im Gegensatz zu diesen drei Altären hat der neue Altar zwei Inschriften. Während Hadrian an einer davon die Epitheten Olympios und Soter hat, steht Zeus Soter an der zweiten Inschrift mit dem Beinamen Sebastos und ist mit einem Blitzbündel dargestellt ${ }^{27}$. Im Vergleich zu anderen drei Altären handelt es sich um einen wesentlichen Unterschied, dass man an der zweiten Inschriften des neuen Altars den Namen Zeus Soter mit einer Darstellung bestimmte. Es

19 Sponsel 2017, 27-32.

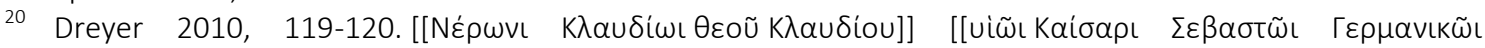

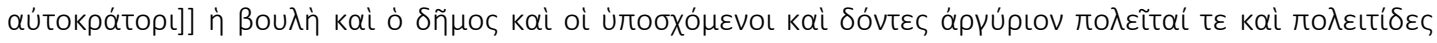

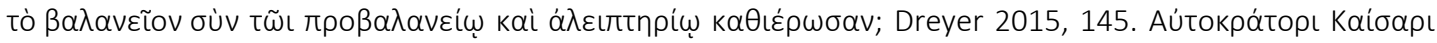

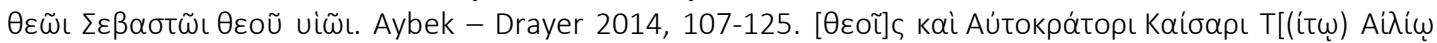

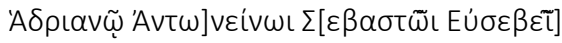

21 Für die Befunde der ähnlichen Kaiserkulte in anderen Städten des westlichen Kleinasiens vgl. Erkelenz 2002, 61-77.

22 Jung 2002, 167ff; Witulski 2007, 90-170, bes. 131 Anm. 528. Für Hadrian mit dem Epitheton Soter s. z. B. IG

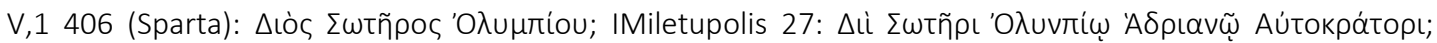

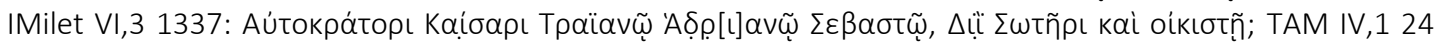

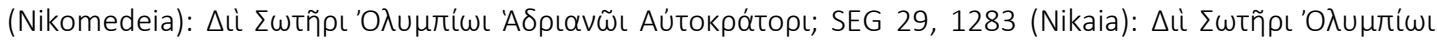

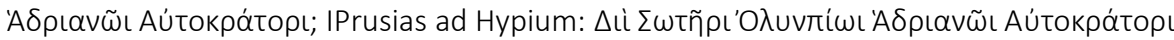

23 IEphesos 3411.

24 Aybek - Gülbay 2018, 1-18.

25 Sponsel 2017, 27-32.

26 Raubitschek 1945, 128-133.

27 Jung 2002, 167. 
sind schon in Metropolis einige Hausaltäre erhalten geblieben, die dem Zeus Soter geweiht wur$\operatorname{den}^{28}$. Aber die Entdeckung einer Inschrift auf einem Altar, die den Namen Hadrian und Zeus Soter beinhalten, betont eine neue Entwicklung ${ }^{29}$.

Obwohl beide Inschriften des neuen Altars in gleicher Zeit bearbeitet wurden, sind der Charakter der Buchstaben unterschiedlich. Es könnte deshalb sein, dass die Darstellung und Inschriften dieses Altars von zwei verschiedenen Steinmetzen ausgeführt wurden. Andererseits auf vielen Altäre, die man in Metropolis fand, steht Zeus Soter niemals mit einem Kaisernamen ${ }^{30}$. Aus diesem Grund kann mit Sicherheit behauptet werden, dass all diese Altäre für Zeus bestimmt wurden. Der wichtigste Punkt auf dem neuen Altar ist die Zusammenstellung der Epitheten Zeus Soter und "Sebastos", die darauf hinweist, dass beide Inschriften zur Verehrung Hadrians und in seiner Lebzeit beschriftet wurde.

\section{Fazit}

Verfolgen wir die Spuren der hadrianischer Zeit in Metropolis, so erhalten wir in der Stadt im Gegensatz zu anderen antiken Städten wie Milet ${ }^{31}$, Ephesos ${ }^{32}$ und Smyrna ${ }^{33}$ eine begrenzte Anzahl von Informationen über diese Zeit. Kaiser Hadrian, bekannt für seine zahlreichen Kult- und Architekturaktivitäten im gesamten mittelmeerischen Raum, wurde auch in Metropolis durch Identifizierung mit Zeus verehrt. Anhand der Altäre ist auch festzustellen, dass seine Gattin Sabina ähnlich wie Hadrian-Zeus mir der Göttin Hera identifiziert und gemeinsam verehrt wurde. Wie in Metropolis ist die Zusammenstellung der Verehrung "Kaiser-Gott" und "KaiserinGöttin" auch in anderen antiken Städten zu treffen ${ }^{34}$. Kaiser Hadrian, der sich mit Göttervater Zeus identifizierte, stützte seine Macht auf den Gott und versuchte es als ein Mittel der Propaganda zu verwenden. Indem Hadrian sich mit Zeus assimiliert ist und sich auf seinen Kult konzentrierte, lehnte die Politik seines Vorgängers der Annexion und der Romanisierung ab und förderte stattdessen die Idee der Koexistenz von griechischen und römischen Traditionen für das Wohlergehen des römischen Reiches ${ }^{35}$. Dazu unternahm und unterstützte er viele Bau- und Sanierungsarbeiten der Zeustempels auch in anderen Städten ${ }^{36}$ des Kleinasiens. Zum Beispiel die Zeustempels in kleinasiatischen Städten, die zum Neokoros aufgestiegen, waren mit hardianischer Zeit eng verbunden ${ }^{37}$. Die Frage, ob in diesen Tempeln nur Hadrian oder der Göttervater verehrt wurde, muss jedoch offen bleiben ${ }^{38}$. Mit Entdeckung der neuen Hausaltäre in Metropolis ist es ersichtlich, dass man den Kaiser mit den Beinamen Olympios und Zeus Soter auch bei den Hausaltären verehrte. Nach dem Ergebnis der in Metropolis durchgeführten Untersuchungen konnte kein Tempel oder Heiligtum mit Hadrian oder mit anderen römischen Kaisern in Verbindung gebracht werden.

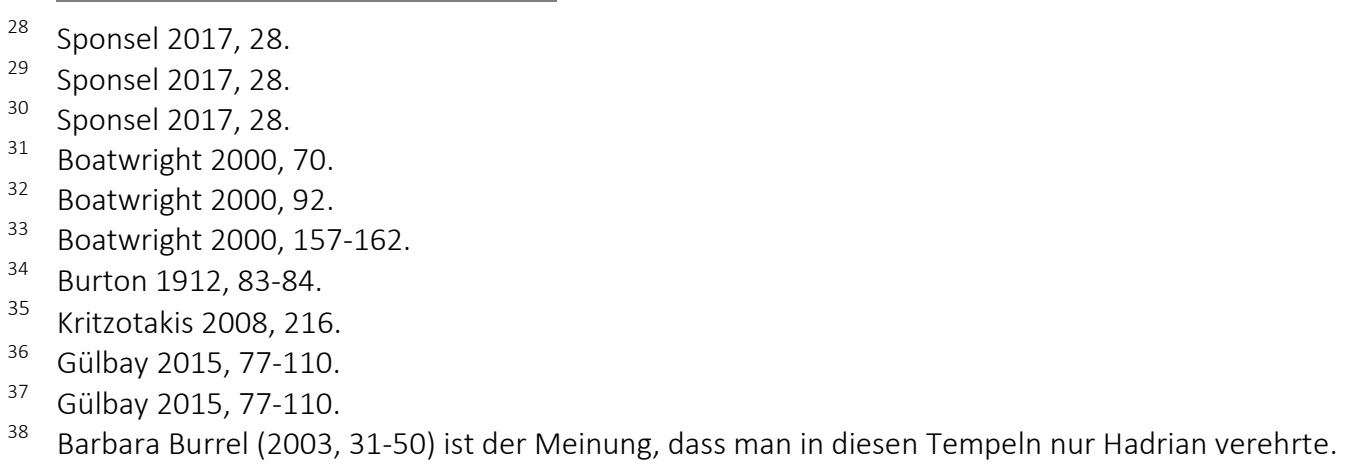




\section{BIBLIOGRAPHIE}

$\mathrm{AE}$

Aybek - Dreyer 2014

L'Année épigraphique. Paris 1888-.

B. Dreyer - S. Aybek, "Ausgrabungen am unteren (Han Yıkığı) römischen Bad und an der Palästra in Metropolis(Ionien): Ein kurzer Bericht mit epigraphischem Anhang". Ed. B. Dreyer, Die Surveys im Hermos- und Kaystrostal und die Grabungen an den Thermen von Metropolis sowie am Stadion von Magnesia am Mäander (Ionien): neue Methoden und Ergebnisse. Berlin (2014) 107-123.

Aybek-Dreyer-Sponsel 2018 S. Aybek, B. Dreyer - C. Sponsel, "Christina Sponsel, Der Kultplatz des Zeus Krezimos in Metropolis in Ionien". Gephyra XV (2018) 71-94.

Aybek - Gülbay 2018 S. Aybek - O. Gülbay, "The Cult of Zeus Krezimos at Metropolis Previous Observations on the Sacred Area and Cult". Byzas XXIV (2018) 1-18.

Baz 1998

Benjamin 1963

Birley 1997

Boatwright 2000

Burrell 2003

Burton 1912

Cadoux 2003

$\mathrm{CIG}$

Dreyer 2010

Dreyer 2015

Erkelenz 2002

Gülbay 2015

Halfmann 1986

IEphesos

IG

IGR

IMilet

F. Baz, Roma Imparator Kültü. Unveröffentlichte Masterarbeit, Marmara Universität. İstanbul 1998.

A. S. Benjamin, "The Altars of Hadrian in Athens and Hadrian's Panhellenic Program". Hesperia XXXII/1 (1963) 57-86.

A. R. Birley, Hadrian: The Restless Emperor. New York, 1997.

M. T. Boatwright, Hadrian and the Cities of the Roman Empire. Princeton 2000.

B. Burrell, "Temples of Hadrian not Zeus". Greek, Roman and Byzantine Studies XLIII (2003) 31-50.

H. F. Burton, "The Worship of the Roman Emperor". Journal of the Biblical World XL/2 (1912) 80-91.

Ce. J. Cadoux, IIlkçağ'da Izmir, Kentin, En Eski Çağlardan I.S. 324'e Kadar Tarihi. İstanbul 2003.

Corpus Inscriptionum Graecarum I-IV, Berlin 1828-1877.

B. Dreyer, "Inschriften aus Metropolis (Ionien)". Eds. S. Aybek - A. K. Öz, Metropolis Ionia II: Yolların Kesiştiği Yer: Recep Meriç Için Yazılar. İstanbul (2010) 115-121.

B. Dreyer, "Eine Landstadt am Puls der Zeit - Neue Inschriften zum Gymnasion und zum Bad aus Metropolis in Ionien". Eds. P. Scholz - D. Wiegandt, Das kaiserzeitliche Gymnasion. Berlin - Boston (2015) 133-148.

D. Erkelenz, "Keine Konkurrenz zum Kaiser. Zur Verleihung der Titel 'Ktistes' und 'Soter' in der römischen Kaiserzeit". Scripta Classica Israelica XXI (2002) 61-77.

O. Gülbay, Anadolu'da Imparator Hadrianus Dönemi Imar Faaliyetleri. İstanbul 2015.

H. Halfmann, Itinera principum. Geschichte und Typologie der Kaiserreisen im römischen Reich, Stuttgart 1986.

C. Börker - R. Merkelbach, IK 15: Die Inschriften von Ephesos [14462000], Bonn 1980.

R. Meriç - R. Merkelbach - J. Nollé - S. Şahin, IK 17.1: Die Inschriften von Ephesos [3001-3500], Bonn 1980.

Inscriptiones Graecae, Berlin 1903-.

R. Cagnat (ed.), Inscriptiones Graecae ad res Romanas pertinentes I-IV, Paris 1906-1927.

Inschriften von Milet: Teil 1. A. Inschriften n. 187-406, hg. von Albert Rehm mit einem Beitrag von Hermann Dessau (Nachdruck), Teil B. Nachträge und Übersetzungen zu den Inschriften n. 1-406 von Peter 
IMilet VI,3

IMiletupolis

|Pergamon III

IPrusias ad Hypium

Jones 1999

Jung 2002

Kienast 1990

Kritzotakis 2008

Mendel 1906

Price 1984

Price 2004

Raubitschek 1945

Robert 1946

SEG

Sponsel 2017

TAM

Tüner Önen 2013

Walton 1957

Witulski 2007

Wörrle 1988
Herrmann, Berlin - New York 1997.

P. Herrmann - G. Wolfgang - E. Norbert, Inschriften von Milet. Teil 3. Inschriften n. 1020-1580. Berlin - New York 2006.

E. Schwertheim, IK 26: Miletupolis. Inschriften und Denkmäler, Bonn 1983.

C. Habicht (ed.), Die Inschriften des Asklepieions. Mit einem Beitrag von Michael Wörrle (Altertümer von Pergamon 8,3), Berlin 1969.

W. Ameling, IK 27: Die Inschriften von Prusias ad Hypium, Bonn 1985.

C. P. Jones, A Decree of Thyateira in Lydia, Chiron 29, 1999, 1-21.

F. Jung, $\Sigma \Omega T H P$ : Studien zur Rezeption eines hellenistischen Ehrentitels im Neuen Testament. Münster 2002.

D. Kienast, Römische Kaisertabelle. Grundzüge einer römischen Kaiserchronologie, Darmstadt ${ }^{5} 1990$.

D. Kritzotakis, Hadrian and the Greek East: Imperial Policy and Communication (unpubl. Diss. Ohia State Uni. 2008).

G. Mendel, Seconde note sur les fouilles exécutées à Aphrodisias par M. Paul Gaudin. Campagne de 1905, CRAI 50, 1906, 158-184.

S. R. F. Price, Gods and Emperors: The Greek Language of the Roman Imperial Cult, JHS 104 (1984) 79-95.

S. R. F. Price, Ritüel ve Iktidar: Küçük Asya'da Roma imparatorluk Kültü. İstanbul, 2004.

A. E. Raubitschek, "Hadrian as the Son of Zeus Eleutherios". American Journal of Archaeology XLIX/2 (1945) 128-133.

L. Robert, Hellenica III. Recueil d'épigraphie, de numismatique et d'antiquités grecques, Paris 1946.

Supplementum Epigraphicum Graecum, Leiden - Amsterdam 1923-.

Ch. Sponsel, Der Areskult in Metropolis (Ionien). Erlangen 2017.

Tituli Asiae Minoris I-V, Wien 1901-1981.

N. Tüner Önen, "Hadrians Reisen im östlichen Mittelmeer anhand neuer Inschriften aus Phaselis". Adalya XVI (2013) 93-106.

F. R. Walton, "Religious Thought in the Age of Hadrian". Numen IV/3 (1957) 165-170.

T. Witulski, Kaiserkult in Kleinasien: Die Entwicklung der kultisch-religiösen Kaiserverehrung in der römischen Provinz Asia. Von Augustus bis Antoninus Pius. Göttingen 2007.

M. Wörrle, Stadt und Fest im kaiserzeitlichen Kleinasien, München 1988. 